gain $(64 \%)$, drowsiness (41\%), nasal congestion (16\%), and dizziness $(16 \%$ )-both postural and non-postural. Prichard and others ${ }^{4}$ reported that methyldopa was unacceptable to $20 \%$ of patients. Side effects are more severe with increased dosage, and tolerance occurs ${ }^{5}$ : a daily requirement of more than $3 \mathrm{~g}$ is, perhaps, an indication for a change in drug rather than for a further increase in dose. ${ }^{6}$

Less common side effects have also been recorded. These include weakness of limbs, blurring of vision, diarrhoea, depression, decrease in mental acuity, Parkinsonism, nocturia, jaundice, drug fever, decreased frequency of sexual intercourse, failure of ejaculation, and non-puerperal lactation. ${ }^{78}$ Some of these effects may result from interference with central adrenergic mechanisms, and there is still controversy about the precise mode of action of methyldopa. Questionnaires ${ }^{8}$ are available to assess the severity and frequency of such symptoms, but their value in measuring problems associated with drug therapy is not established. When symptoms really interfere with everyday life alternative therapy should be considered. Church has reported drug rashes in patients on methyldopa ${ }^{9}$ - urticaria and nummular, palmar, and discoid eczema occur, particularly in individuals with previous skin disorders. A positive Coombs test has been reported ${ }^{10}$ in $20 \%$ of patients on methyldopa for more than six months, and $0.2 \%$ of these patients may develop a symptomatic haemolytic anaemia, ${ }^{11}$ while others may more rarely develop reversible leucopenia ${ }^{12}$ or thrombocytopenia. ${ }^{13}$ Such patients should stop methyldopa therapy, but the advice that should be given to patients found to have antinuclear or rheumatoid factors is less clear.

${ }^{1}$ Silverberg, D. S., et al., Canadian Medical Association fournal, 1974, 111, 769.

2 Oates, J. A., et al., Science, 1960, 131, 1890.

3 Johnson, P., et al., British Medical fournal, 1966, 1, 133.

4 Prichard, B. N. C., et al., British Medical fournal, 1968, 1, 135.

5 Smirk, H., British Medical fournal, 1963, 1, 146.

6 Bulpitt, C. J., and Dollery, C. T., Practitioner, 1971, 207, 43.

7 Bulpitt, C. J., and Dollery, C. T., Practitioner, 1971, 207, 43. 29.

${ }^{7}$ Beyler, L., and Herxheimer, A., Side Effects of Drugs, 1972, 7, 299.

${ }^{8}$ Bulpitt, C. J., and Dollery, C. T., British Medical fournal,

${ }^{9}$ Church, R., British Fournal of Dermatology, 1974, 91, 373.

11 Worlledge, S. M., Carstairs, K. C., and Dacie, J. V., Lancet, 1966, 2, 135

13 Benraad, A. H., and Schoenaker, A. H., Lancet, 1965, 2, 292.

\section{Undiagnosed Haematuria}

Anyone with experience of medical records will be familiar with cases of haematuria in which, despite thorough investigation, no specific cause can be identified. Though in some patients the clinical features may point to inflammation or congestion of a particular organ such as the kidney, bladder, or prostate, others without accompanying symptoms may have to be consigned at least temporarily to a diagnostic limbo characterized by such evocative descriptions as "essential haematuria" or "urothelial epistaxis." Such cases impose a special responsibility on the clinician, especially since bleeding into the urinary tract is so often the first indication of serious or malignant disease.

In a recent survey of 64 cases from the University Hospital of South Manchester ${ }^{1}$ it was suggested that between 5 and $10 \%$ of patients presenting with haematuria remain undiagnosed after initial investigation. This estimate is similar to statistics from Texas, ${ }^{2}$ where 25 out of 237 patients fell into the "unknown origin" category. Much undoubtedly depends on the standard of urological expertise as well as on the supporting diagnostic facilities, but there seems little reason for assuming that this ratio represents an underestimate.

In most cases presenting with overt haematuria the cause can be established without undue difficulty, but it is important that investigation should always proceed on regular lines. Initial inquiry should be made about the quality and duration of the bleeding as well as any accompanying symptoms or evidence of haemorrhage from other sites. Age, sex, race, and geographical factors must likewise be taken into account. Totally blood stained urine, especially if painless, should always arouse suspicion of a serious underlying lesion, while any associated features may help in suggesting its nature and extent. In obscure cases the possibility of medical conditions such as nephritis, systemic blood disorders, and hypertension have to be considered, and exposure to certain drugs, particularly anticoagulants, must be excluded.

Routine investigation should include both microscopic and bacteriological examination of the urine to confirm the presence of red blood cells and identify any accompanying infection. In some cases cytological examination of the urinary deposit may also be appropriate in a search for malignant cells. Intravenous urography is almost mandatory, unless excluded by serious allergy or other disease, and may prove valuable not only in establishing the cause of bleeding but in demonstrating any coexistent structural or functional changes in the urinary tract. Endoscopy, combined with pelvic examination under anaesthesia, is especially useful in cases where haematuria has been recent or profuse; this investigation not only will determine the nature and extent of local disease but may exclude other intercurrent conditions such as prostatic hypertrophy. In this respect the accuracy of cystoscopic diagnosis and biopsy has recently been transformed by the introduction of fibreglass illumination, and such equipment should now be regarded as essential in modern urological departments. Other more complex methods of examination by retrograde or prograde urography under the $x$-ray intensifier screen, as well as by renal angiography, may be required to identify lesions in the upper urinary tract, while the application of ultrasonic diagnosis serves a useful purpose in distinguishing between renal tumours and cysts.

Analysis of the Manchester series, covering a period of $4 \frac{1}{2}$ years, showed that routine examination was generally successful in the detection of significant urological disease. Of the 64 remaining patients in whom early positive diagnosis was unattainable, 12 ( 8 men and 4 women) had associated hypertension and 14 (10 men and 4 women) had symptoms suggestive of non-specific infection. Six had had symptoms of prostatism and three had been on anticoagulants. There were no associated features in 22 , and the residue fell into individual categories in which such causes as local congestion due to intercourse, heavy drinking, or athletic exercise were postulated. In a subsequent follow-up study there were only three cases in which serious disease became apparent: one hypernephroma, one carcinoma of the renal pelvis, and one patient with sickle-cell trait. Nevertheless such examples are sufficient to emphasize the need for further review in all cases of undiagnosed bleeding, particularly in relation to obscure renal lesions and systemic blood diseases. Even when initial investigation has suggested a local congestive or inflammatory cause the possibility of a coexistent neoplasm or a specific infection such as tuberculosis must still be borne in mind, and further studies are warranted.

\footnotetext{
1 O'Reilly, P. H., Postgraduate Medical fournal, 1974, 50, 746.
2 Burkholder, G. V., et al., Fournal of the American Medical Association, $1969,210,1729$.
} 